\title{
Bounding the Higgs Width Using Effective Field Theory*
}

\section{Margherita Ghezzi, Giampiero Passarino ${ }^{\dagger}$ and Sandro Uccirati}

Dipartimento di Fisica Teorica, Università di Torino, INFN, Sezione di Torino, Italy

E-mail: mghezzi@to.infn.it, giampiero@to.infn.it, uccirati@to.infn.it

An interesting question is how present and future experiments will be able to probe the couplings of the Higgs boson and its intrinsic width at a high level of precision. There is a wide variety of beyond the Standard Model (BSM) theories where the Higgs couplings differ from the Standard Model (SM) ones by less that $10 \%$. We take the SM as the theory of "light" degrees of freedom, i.e. $d=4$ operators and simulate the unknown extension of the SM by the most general set of $d=6$ operators. In particular we provide an explicit example of momentum-dependent modification of Higgs couplings.

Loops and Legs in Quantum Field Theory

27 April 2014 - 02 May 2014

Weimar, Germany

*Work supported by MIUR under contract 2001023713_006, by UniTo - Compagnia di San Paolo under contract ORTO11TPXK and by the Executive Research Agency (REA) of the European Union under the Grant Agreement PITNGA-2012-316704 (HiggsTools).

† Speaker. 


\section{Introduction}

Indirect constraints on the total Higgs width at LHC have received considerable attention and the CMS Collaboration [1] has presented the first measurement. The analysis is based on correlating the Higgs signal strength with measurements in the off-shell region. In Refs. [2, 3] the off-shell production cross section has been shown to be sizeable at high $\mathrm{ZZ}$-invariant mass in the gluon fusion production mode, with a ratio relative to the on-peak cross section of the order of $8 \%$ at a center-of-mass energy of $8 \mathrm{TeV}$. This ratio can be enhanced up to about $20 \%$ when a kinematical selection used to extract the signal in the resonant region is taken into account [4]. This arises from the vicinity of the on-shell $\mathrm{Z}$ pair production threshold, and is further enhanced at the on-shell top pair production threshold.

In Refs. [5] the authors demonstrated that, with few assumptions and using events with pairs of $\mathrm{Z}$ particles, the high invariant mass tail can be used to constrain the Higgs width.

This note introduces the bases for a model-independent interpretation of the constraint, generalizing the arguments given in Ref. [6]; for complementary studies see Refs. [7, 8, 9].

\section{On-shell $\infty$-degeneracy}

Refs. $[10,5,11,12]$ consider the following scenario (on-shell $\infty$-degeneracy): allow for a scaling of the Higgs couplings $\left(g_{i}, g_{f}\right)$ and of the total Higgs width $\left(\gamma_{\mathrm{H}}\right)$ defined by

$$
\sigma_{i \rightarrow \mathrm{H} \rightarrow f}=(\sigma \cdot \mathrm{BR})=\frac{\sigma_{i}^{\mathrm{prod}} \Gamma_{f}}{\gamma_{\mathrm{H}}}, \quad \sigma_{i \rightarrow \mathrm{H} \rightarrow f} \propto \frac{g_{i}^{2} g_{f}^{2}}{\gamma_{\mathrm{H}}}, \quad g_{i, f}=\xi g_{i, f}^{\mathrm{SM}}, \quad \gamma_{\mathrm{H}}=\xi^{4} \gamma_{\mathrm{H}}^{\mathrm{SM}} .
$$

Looking for $\xi$-dependent effects in the highly off-shell region is an approach that raises sharp questions on the nature of the underlying extension of the SM; furthermore it does not take into account variations in the SM background and the signal strength in 41, relative to the expectation for the SM Higgs boson, is measured by CMS to be $0.91_{-0.24}^{+0.30}$ [13] and by ATLAS to be $1.43_{-0.35}^{+0.40}$ [14]. We adopt the approach of Ref. [15] (in particular Eqs. (1-18)) which are based on the $\kappa$-language, allowing for a consistent "Higgs Effective Field Theory" (HEFT) interpretation, see Ref. [16]. For example, neglecting loop-induced vertices, in the production via gluon fusion we have:

$$
\kappa_{\mathrm{g}}^{2}=\frac{\sigma_{\mathrm{ggH}}\left(M_{\mathrm{H}}\right)}{\sigma_{\mathrm{ggH}}^{\mathrm{SM}}\left(M_{\mathrm{H}}\right)}=\frac{\kappa_{\mathrm{t}}^{2} \cdot \sigma_{\mathrm{ggH}}^{\mathrm{tt}}\left(M_{\mathrm{H}}\right)+\kappa_{\mathrm{b}}^{2} \cdot \sigma_{\mathrm{ggH}}^{\mathrm{bb}}\left(M_{\mathrm{H}}\right)+\kappa_{\mathrm{t}} \kappa_{\mathrm{b}} \cdot \sigma_{\mathrm{ggH}}^{\mathrm{tb}}\left(M_{\mathrm{H}}\right)}{\sigma_{\mathrm{ggH}}^{\mathrm{tt}}\left(M_{\mathrm{H}}\right)+\sigma_{\mathrm{ggH}}^{\mathrm{bb}}\left(M_{\mathrm{H}}\right)+\sigma_{\mathrm{ggH}}^{\mathrm{tb}}\left(M_{\mathrm{H}}\right)} .
$$

The measure of off-shell effects can be interpreted as a constraint on $\gamma_{\mathrm{H}}$ only when we scale couplings and total width according to Eq.(2.1) to keep $\sigma_{\text {peak }}$ untouched, although its value is known with 15-20\% accuracy. The generalization of Eq.(2.1) is an $\infty^{2}$-degeneracy, $\kappa_{i} \kappa_{f}=\kappa_{\mathrm{H}}$, where $g_{i, f}=\kappa_{i, f} g_{i, f}^{S M}, \gamma_{\mathrm{H}}=\kappa_{\mathrm{H}}^{2} \gamma_{\mathrm{H}}^{\mathrm{SM}}$.

On the whole, we have a constraint in the multidimensional $\kappa$-space of rescaling factors for couplings (for gluon fusion we have for istance, $\kappa_{i}=\kappa_{\mathrm{g}}\left(\kappa_{\mathrm{t}}, \kappa_{\mathrm{b}}\right)$ ). Only on the assumption of degeneracy we can prove that off-shell effects "measure" $\kappa_{\mathrm{H}}$; a combination of on-shell effects (measuring $\kappa_{i} \kappa_{f} / \kappa_{\mathrm{H}}$ ) and off-shell effects (measuring $\kappa_{i} \kappa_{f}$ ) gives information on $\kappa_{\mathrm{H}}$ without prejudices. Denoting by $S$ the signal and by $I$ the interference and assuming that $I_{\text {peak }}$ is negligible we have

$$
\frac{\mathrm{S}_{\text {off }}}{\mathrm{S}_{\text {peak }}} \kappa_{\mathrm{H}}^{2}+\frac{\mathrm{I}_{\mathrm{off}}}{\mathrm{S}_{\text {peak }}} \frac{\kappa_{\mathrm{H}}}{x_{i f}}, \quad x_{i f}=\frac{\kappa_{i} \kappa_{f}}{\kappa_{\mathrm{H}}},
$$


for the normalized $\mathrm{S}+\mathrm{I}$ off-shell cross section. The background, e.g. $\mathrm{gg} \rightarrow 41$, is also changed by the inclusion of $d=6$ operators and one cannot claim that New Physics is modifying only the signal.

\section{Higgs Effective Field Theory}

CMS results raise a question: is there a QFT behind degeneracy with a consistent BSM interpretation? Our starting point is the following Lagrangian

$$
\mathscr{L}=\mathscr{L}_{4}+\sum_{n>4} \sum_{i=1}^{N_{n}} \frac{a_{i}^{n}}{\Lambda^{n-4}} \mathscr{O}_{i}^{(d=n)}
$$

where $\mathscr{L}_{4}$ is the Standard Model (SM) and $\Lambda$ is a given cut-off. Any (pseudo-)observable starting at $\mathrm{O}\left(g^{\mathrm{N}}\right)$ is given by

$$
\mathrm{A}=\sum_{n=\mathrm{N}}^{\infty} \sum_{l=0}^{n} \sum_{k=1}^{\infty} g^{n} g_{4+2 k}^{l} \mathrm{~A}_{n l k}, \quad g_{4+2 k}=1 /\left(\sqrt{2} G_{\mathrm{F}} \Lambda^{2}\right)^{k},
$$

where no hierarchy of higher-dimensional operators is assumed; for $\operatorname{dim}=6$ operators we follow the work of Ref. [17] (for alternative approaches see Ref. [18] and also Ref. [19]). According to the work of Ref. [20] (see also Ref. [21]) we distinguish between potentially-tree-generated (PTG) operators and loop-generated (LG) operators (an operator is PTG if it is generated in at least one extension of SM). It can be argued that (at LO) the basis operator should be chosen from among the PTG operators but it is also evident that one can take an $\mathscr{O}_{\mathrm{LG}}^{(6)}$ and contract two lines forming a loop, which requires renormalization of some $\mathscr{O}^{(4)}$ and a SM vertex with $\mathscr{O}_{\mathrm{PTG}}^{(6)}$ is also required. Furthermore, if we assume that the high-energy theory is weakly-coupled and renormalizable it follows that the PTG/LG classification of Ref. [20] (used here) is correct. If we do not assume the above but work always in some EFT context (i.e.. also the next high-energy theory is EFT, possibly involving some strongly interacting theory) then classification changes, see Eqs. (A1-A2) of Ref. [22]. Decoupling is also assumed, e.g. colored scalars disappear from the low energy physics as their mass increases but the same is not true for fermions.

Furthermore, we will not address the question of constraints on electroweak effective operators $[7,23,8]$; penalty functions can always be added in the fit.

Phrased differently, our questions are the following: are the $g_{i, f}$ factors in Eq.(2.1) constant or running? What is their relation with the $\kappa$-language once we extend it to next-to-leading (NLO) order? What is their relation with the Wilson coefficients of the relevant operators? What can we learn, in a model-independent way (although supporting a weakly-coupled and renormalizable UV completion), from off-shell cross section measurements?

Before we provide an answer we would like to stress that there are two ways of formulating an effective field theory [24]: a) mass-dependent scheme(s) or Wilsonian EFT, b) mass-independent scheme(s) or continuum EFT (CEFT). Only a) is conceptually consistent with the image of an EFT as a low-energy approximation to a high-energy theory, however inclusion of NLO corrections is only meaningful in b) since we cannot regularize with a cut-off and NLO requires regularization.

There is an additional problem, CEFT requires evolving our theory to lower scales until we get below the "heavy-mass" scale where we use $\mathscr{L}=\mathscr{L}_{\text {sM }}+d \mathscr{L}, d \mathscr{L}$ encoding matching corrections 
at the boundary. Therefore, CEFT does not integrate out heavy degrees of freedom but removes them compensating for by an appropriate matching calculation. From this point of view HEFT is not quite the same as it is usually discussed since we have no theory approaching the boundary from above (cf. low-energy SM, weak effects on $g-2$ etc.).

\subsection{Renormalization}

Once we have the Lagrangian of Eq.(3.1) the whole renormalization procedure (see Refs. [25, $26,27]$ must be reinitialized. Thus, part of the procedure consists of several steps:

- evaluation of tadpoles and introduction of counterterms, $\Phi=Z_{\phi}^{1 / 2} \Phi_{\mathrm{R}}$ etc., where

$$
\mathrm{Z}_{\phi}=1+\frac{g^{2}}{16 \pi^{2}}\left(\delta Z_{\phi}^{(4)}+g_{6} \delta Z_{\phi}^{(6)}\right) \frac{1}{\bar{\varepsilon}}
$$

- self-energies are computed and counterterms fixed to make them ultraviolet $\mathscr{O}^{(4)}, \mathscr{O}^{(6)}$-finite;

- $\mu$-decay is computed and coupling constant renormalization follows: $g \rightarrow g_{\mathrm{R}}$;

- furthermore, finite renormalization is performed, e.g.

$$
M_{\mathrm{R}}^{2}=M_{\mathrm{W}}^{2}\left[1+\frac{g_{\mathrm{R}}^{2}}{16 \pi^{2}}\left(\operatorname{Re} \Sigma_{\mathrm{WW}}-\delta \mathrm{Z}_{\mathrm{M}}\right)\right], \quad \text { etc.; }
$$

- Dyson re-summed propagators are finite; for instance

$$
\begin{aligned}
\Delta_{\mathrm{H}}^{-1} & =\mathrm{Z}_{\mathrm{H}}\left(-s+\mathrm{Z}_{m_{\mathrm{H}}} M_{\mathrm{H}}^{2}\right)-\frac{1}{(2 \pi)^{4} i} \Sigma_{\mathrm{HH}}, \\
m_{\mathrm{H}}^{2} & =M_{\mathrm{H}}^{2}\left[1+\frac{g_{\mathrm{R}}^{2}}{16 \pi^{2}}\left(\mathrm{~d} M_{\mathrm{H}}^{(4)}+g_{6} \mathrm{~d} M_{\mathrm{H}}^{(6)}\right)\right],
\end{aligned}
$$

where $m_{\mathrm{H}}$ is the renormalized Higgs mass and $M_{\mathrm{H}}$ is the on-shell mass (in this note we are not going to discuss/introduce complex poles).

\subsection{Effective couplings}

Consider off-shell gluon-gluon fusion ( $\mathrm{gg} \rightarrow \mathrm{H}$, where $v_{\mathrm{H}}$ is the Higgs virtuality): it requires the introduction of renormalization factors $\mathrm{Z}_{\mathrm{H}}, \mathrm{Z}_{\mathrm{g}}$ for the external fields, $\mathrm{Z}_{g}$ for the $S U(2)$ coupling and $\mathrm{Z}_{g_{\mathrm{S}}}$ for the strong coupling constant. The amplitude is obviously $\mathscr{O}^{(4)}$-finite but not $\mathscr{O}^{(6)}$-finite and involves the following Wilson coefficients (see Ref. [17]): $a_{\phi \mathrm{D}}, a_{\phi \square}, a_{\mathrm{t} \phi}, a_{\mathrm{b} \phi}$ for PTG operators and $a_{\mathrm{\phi W}}, a_{\phi \mathrm{g}}, a_{\mathrm{tg}}, a_{\mathrm{bg}}$ for LG operators. It is convenient to introduce

$$
\begin{gathered}
a_{\mathrm{tg}}=\mathrm{W}_{1}, \quad a_{\mathrm{bg}}=\mathrm{W}_{2}, \quad a_{\phi \mathrm{g}}=\mathrm{W}_{3}, \\
a_{\mathrm{b \phi}}+\frac{1}{4} a_{\phi \mathrm{D}}-a_{\Phi \mathrm{W}}-a_{\phi \square}=\mathrm{W}_{4}, \quad a_{\mathrm{t \phi}}-\frac{1}{4} a_{\phi \mathrm{D}}+a_{\Phi \mathrm{W}}+a_{\phi \square}=\mathrm{W}_{5} .
\end{gathered}
$$

$\mathscr{O}^{(6)}$-finiteness requires extra renormalization, i.e.

$$
\mathrm{W}_{i}=\sum_{j} Z_{i j}^{\mathrm{mix}} W_{j}^{\mathrm{R}}\left(\mu_{\mathrm{R}}\right), \quad Z_{i j}^{\mathrm{mix}}=\delta_{i j}+\frac{g g_{\mathrm{S}}}{16 \pi^{2}} \delta Z_{i j}^{\text {mix }} \frac{1}{\bar{\varepsilon}}, \quad \delta Z_{31(2)}^{\text {mix }}=-\frac{1}{2 \sqrt{2}} \frac{M_{\mathrm{t}(\mathrm{b})}}{M_{\mathrm{W}}} .
$$


We define building blocks using $B_{0}\left(C_{0}\right)$ for the scalar two(three)-point function

$$
\begin{gathered}
\frac{8 \pi^{2}}{i g_{\mathrm{S}}^{2}} \frac{M_{\mathrm{W}}}{M_{\mathrm{q}}^{2}} \mathrm{~A}_{\mathrm{q}}^{\mathrm{LO}}=2-\left(4 M_{\mathrm{q}}^{2}-v_{\mathrm{H}}\right) C_{0}\left(-v_{\mathrm{H}}, 0,0 ; M_{\mathrm{q}}, M_{\mathrm{q}}, M_{\mathrm{q}}\right), \\
\frac{32 \pi^{2}}{i g_{\mathrm{S}}^{2}} \frac{M_{\mathrm{W}}^{2}}{M_{\mathrm{q}}} \mathrm{A}_{\mathrm{q}}^{\mathrm{nfc}}=8 M_{\mathrm{q}}^{4} C_{0}\left(-v_{\mathrm{H}}, 0,0 ; M_{\mathrm{q}}, M_{\mathrm{q}}, M_{\mathrm{q}}\right)+v_{\mathrm{H}}\left[1-B_{0}\left(-v_{\mathrm{H}} ; M_{\mathrm{q}}, M_{\mathrm{q}}\right)\right]-4 M_{\mathrm{q}}^{2},
\end{gathered}
$$

and process dependent $\kappa$-factors (which are now linear combinations of Wilson coefficients)

$$
\kappa_{\mathrm{b}}=1+g_{6}\left[\frac{1}{2} \frac{M_{\mathrm{b}}}{M_{\mathrm{W}}} W_{2}^{\mathrm{R}}-\frac{1}{\sqrt{2}} W_{4}^{\mathrm{R}}\right], \quad \kappa_{\mathrm{t}}=1+g_{6}\left[\frac{1}{2} \frac{M_{\mathrm{t}}}{M_{\mathrm{W}}} W_{1}^{\mathrm{R}}-\frac{1}{\sqrt{2}} W_{5}^{\mathrm{R}}\right] .
$$

With their help we construct the full $4+6$ amplitude for $g g \rightarrow \mathrm{H}$,

$$
\mathrm{A}_{\mathrm{gg} \rightarrow \mathrm{H}}^{(4+6)}=g \sum_{\mathrm{q}=\mathrm{b}, \mathrm{t}} \kappa_{\mathrm{q}} \mathrm{A}_{\mathrm{q}}^{\mathrm{LO}}+i \frac{g_{6} g_{\mathrm{S}}}{\sqrt{2}} \frac{M_{\mathrm{H}}^{2}}{M_{\mathrm{W}}} W_{3}^{\mathrm{R}}+g_{6} g\left[W_{1}^{\mathrm{R}} \mathrm{A}_{\mathrm{t}}^{\mathrm{nfc}}+W_{2}^{\mathrm{R}} \mathrm{A}_{\mathrm{b}}^{\mathrm{nfc}}\right],
$$

and derive a true relation expressing deviations from the SM and momentum-dependent modification of Higgs couplings,

$$
\mathrm{A}^{(4+6)}(\mathrm{gg} \rightarrow \mathrm{H})=\xi_{\mathrm{g}}\left(v_{\mathrm{H}}\right) \mathrm{A}^{(4)}(\mathrm{gg} \rightarrow \mathrm{H}) .
$$

Therefore, the answer to the question on the nature of the couplings in Eq.(2.1) is that the effective (running) scaling-factor $\xi_{i}$ is not a $\kappa$ (constant) parameter unless we put $\mathscr{O}_{\mathrm{LG}}^{(6)}=0$ and $\kappa_{\mathrm{b}}=\kappa_{\mathrm{t}}$.

\subsection{Scale dependence}

The $\left(\mu_{\mathrm{R}}\right)$ scale dependence of the full amplitude (from the point of view of renormalization group evolution of the SM dim $=6$ operators see also Refs. [28, 29, 30, 31]) follows from the fact that we have no matching condition. Therefore the mixing among Wilson coefficients should be rewritten as

$$
\begin{gathered}
\mathrm{W}_{i}=\sum_{j} Z_{i j}^{\mathrm{mix}} W_{j}^{\mathrm{R}}\left(\mu_{\mathrm{R}}\right), \quad \mathrm{W}_{1}=a_{\gamma \gamma}=s_{\theta} c_{\theta} a_{\Phi \mathrm{WB}}+c_{\theta}^{2} a_{\phi \mathrm{B}}+s_{\theta}^{2} a_{\phi \mathrm{W}}, \quad \text { etc. } \\
Z_{i j}^{\text {mix }}=\delta_{i j}+\frac{g_{\mathrm{R}}^{2}}{16 \pi^{2}}\left[\delta Z_{i j}^{\text {mix }} \frac{1}{\bar{\varepsilon}}+\Delta_{i j} \ln \frac{M_{\mathrm{H}}^{2}}{\mu_{\mathrm{R}}^{2}}\right] \\
M_{\mathrm{W}}^{2} \Delta_{11}=\frac{1}{4}\left[8 s_{\theta}^{2}\left(2 s_{\theta}^{2}-c_{\theta}^{2}\right) M_{\mathrm{W}}^{2}+\left(4 s_{\theta}^{2} c_{\theta}^{2}-5\right) M_{\mathrm{H}}^{2}\right], \quad \text { etc. }
\end{gathered}
$$

Here $c_{\theta}^{2}=M_{\mathrm{W}}^{2} / M_{\mathrm{Z}}^{2}$. In the $\overline{\mathrm{MS}}$ scheme this defines $\mu_{\mathrm{R}}$-dependent renormalized coefficients. The life and death of $\mu_{\mathrm{R}}$ can be summarized as follows: consider the $\gamma$ bare propagator

$$
\begin{gathered}
\Delta_{\gamma}^{-1}=-s-\frac{g^{2}}{16 \pi^{2}} \Sigma_{\gamma \gamma}(s), \quad\{\mathscr{X}\}=\left\{s, m^{2}, m_{0}^{2}, m_{\mathrm{H}}^{2}, m_{\mathrm{t}}^{2}, m_{\mathrm{b}}^{2}\right\}, \\
\Sigma_{\gamma \gamma}(s)=\left(\mathrm{D}^{(4)}+g_{6} \mathrm{D}^{(6)}\right) \frac{1}{\bar{\varepsilon}}+\sum_{x \in \mathscr{X}}\left(\mathrm{L}_{x}^{(4)}+g_{6} \mathrm{~L}_{x}^{(6)}\right) \ln \frac{x}{\mu_{\mathrm{R}}^{2}}+\Sigma_{\gamma \gamma}^{\mathrm{rest}} .
\end{gathered}
$$

Build the $\gamma$ renormalized propagator

$$
\left.\Delta_{\gamma}^{-1}\right|_{\text {ren }}=-Z_{\gamma} s-\frac{g^{2}}{16 \pi^{2}} \Sigma_{\gamma \gamma}(s)=-s-\frac{g^{2}}{16 \pi^{2}} \Sigma_{\gamma \gamma}^{\mathrm{ren}}(s),
$$


and the renormalized $\gamma$ self-energy

$$
\Sigma_{\gamma \gamma}^{\mathrm{ren}}(s)=\sum_{x \in \mathscr{X}}\left(\mathrm{L}_{x}^{(4)}+g_{6} \mathrm{~L}_{x}^{(6)}\right) \ln \frac{x}{\mu_{\mathrm{R}}^{2}}+\Sigma_{\gamma \gamma}^{\mathrm{rest}} .
$$

After finite renormalization we obtain

$$
\Sigma_{\gamma \gamma}^{\mathrm{ren}}(s)=\Pi_{\gamma \gamma}^{\mathrm{ren}}(s) s, \quad \frac{\partial}{\partial \mu_{\mathrm{R}}}\left[\Pi_{\gamma \gamma}^{\mathrm{ren}}(s)-\Pi_{\gamma \gamma}^{\mathrm{ren}}(0)\right]=0,
$$

including $\mathscr{O}^{(6)}$ contribution. Thus, there is no $\mu_{\mathrm{R}}$-problem when a subtraction point is available (e.g. $q^{2}=0$ for the electric charge).

\subsection{Complexity and Background}

The example of $\mathrm{gg} \rightarrow \mathrm{H}$ is particularly simple but there is an increasing degree of complexity when we move to other processes. For instance, for $\mathrm{H} \rightarrow \gamma \gamma$ we have $3 \mathrm{LO}$ amplitudes $\left(\mathrm{A}_{\mathrm{t}}^{\mathrm{LO}}, \mathrm{A}_{\mathrm{b}}^{\mathrm{LO}}, \mathrm{A}_{\mathrm{W}}^{\mathrm{LO}}\right), 3 \kappa$-factors and 6 Wilson coefficients \& non-factorizable amplitudes. For $\mathrm{H} \rightarrow \mathrm{ZZ}$ there is $1 \mathrm{LO}$ amplitude, $6 \mathrm{NLO}$ amplitudes, $6 \mathrm{\kappa}$-factors

$$
\delta^{\mu v} \sum_{i=\mathrm{t}, \mathrm{b}, \mathrm{B}} \mathrm{A}_{i, \mathrm{D}}^{\mathrm{NLO}}+p_{2}^{\mu} p_{1}^{v} \sum_{i=\mathrm{t}, \mathrm{b}, \mathrm{B}} \mathrm{A}_{i, \mathrm{P}}^{\mathrm{NLO}}
$$

and 16 Wilson coefficients \& non-factorizable amplitudes, etc..

Finally, we consider the background, e.g. $\bar{u} \mathrm{u} \rightarrow \mathrm{ZZ}$. The following combinations of Wilson coefficients appear:

$$
\begin{aligned}
& \mathrm{W}_{1}=a_{\gamma \gamma}=s_{\theta} c_{\theta} a_{\Phi \mathrm{WB}}+c_{\theta}^{2} a_{\phi \mathrm{B}}+s_{\theta}^{2} a_{\phi \mathrm{W}} \\
& \mathrm{W}_{2}=a_{\mathrm{ZZ}}=-s_{\theta} c_{\theta} a_{\Phi \mathrm{WB}}+s_{\theta}^{2} a_{\phi \mathrm{B}}+c_{\theta}^{2} a_{\phi \mathrm{W}} \\
& \mathrm{W}_{3}=a_{\gamma \mathrm{Z}}=2 s_{\theta} c_{\theta}\left(a_{\phi \mathrm{W}}-a_{\phi \mathrm{B}}\right)+\left(c_{\theta}^{2}-s_{\theta}^{2}\right) a_{\Phi \mathrm{WB}} \\
& \mathrm{W}_{4}=a_{\phi \mathrm{D}}, \quad \mathrm{W}_{5}=a_{\phi \mathrm{q}}^{(3)}+a_{\phi \mathrm{q}}^{(1)}-a_{\phi \mathrm{u}}, \quad \mathrm{W}_{6}=a_{\phi \mathrm{q}}^{(3)}+a_{\phi \mathrm{q}}^{(1)}+a_{\phi \mathrm{u}} .
\end{aligned}
$$

Defining the kinematical part of the LO amplitude as

$$
\mathrm{A}^{\mathrm{LO}}=\frac{M_{\mathrm{Z}}^{4}}{t^{2}}+\frac{M_{\mathrm{Z}}^{4}}{u^{2}}-\frac{t}{u}-\frac{u}{t}-4 \frac{M_{\mathrm{Z}}^{2} s}{t u},
$$

we obtain the result $(\overline{\mathrm{u}} \mathrm{u} \rightarrow \mathrm{ZZ})$

$$
\sum_{\text {spin }}\left|\mathrm{A}^{(4+6)}\right|^{2}=g^{4} \mathrm{~A}^{\mathrm{LO}}\left[\mathrm{F}^{\mathrm{LO}}\left(s_{\theta}\right)+\frac{g_{6}}{\sqrt{2}} \sum_{i=1}^{6} \mathrm{~F}^{i}\left(s_{\theta}\right) \mathrm{W}_{i}\right] .
$$

\section{Conclusions}

Thanks to the work of different groups we know that a combination of on-shell effects and off-shell effects gives information on the Higgs boson intrinsic width. Interpretation of the measurements and possible signals for deviations from the SM cannot live without an underlying theory. We have shown that, within a model-independent NLO approach, the Higgs couplings must 
be interpreted as "running" couplings, expressible as linear combinations of Wilson coefficients of higher-dimensional operators and including non-factorizable components. Consequently any measurement of the couplings can be interpreted as a measure of the Wilson coefficients. Assuming that LHC will reach the needed sensitivity, this information will be a (blurred) arrow in the space of BSM Lagrangians, and we should simply focus the arrow.

It is worth noting that this question is highly difficult to receive a complete answer at the LHC. The main goal will be to identify the structure of the effective Lagrangian and to derive qualitative information on new physics; the question of the ultraviolet completion cannot be answered unless there is sensitivity to $\mathrm{d}>6$ operators. Therefore, we are proposing a relatively modest goal on the road to understand if the effective theory can be UV completed (bottom-up approach with no obvious embedding).

\section{References}

[1] CMS Collaboration, V. Khachatryan et al., Constraints on the Higgs boson width from off-shell production and decay to Z-boson pairs, arXiv:1405.3455 [hep-ex] .

[2] N. Kauer and G. Passarino, Inadequacy of zero-width approximation for a light Higgs boson signal, JHEP 1208 (2012) 116, arXiv: 1206.4803 [hep-ph] .

[3] G. Passarino, Higgs Interference Effects in $g g \rightarrow$ ZZ and their Uncertainty, JHEP 1208 (2012) 146, arXiv:1206.3824 [hep-ph].

[4] N. Kauer, Inadequacy of zero-width approximation for a light Higgs boson signal, Mod.Phys.Lett. A28 (2013) 1330015, arXiv: 1305.2092 [hep-ph] .

[5] F. Caola and K. Melnikov, Constraining the Higgs boson width with ZZ production at the LHC, Phys.Rev. D88 (2013) 054024, arXiv: 1307.4935 [hep-ph] .

[6] G. Passarino, Higgs CAT, arXiv:1312.2397 [hep-ph] .

[7] H. Mebane, N. Greiner, C. Zhang, and S. Willenbrock, Constraints on Electroweak Effective Operators at One Loop, Phys.Rev. D88 (2013) no. 1, 015028, arXiv: 1306.3380 [hep-ph] .

[8] C. Englert, A. Freitas, M. Muhlleitner, T. Plehn, M. Rauch, et al., Precision Measurements of Higgs Couplings: Implications for New Physics Scales, arXiv:1403.7191 [hep-ph] .

[9] C. Englert and M. Spannowsky, Limitations and Opportunities of Off-Shell Coupling Measurements, arXiv:1405.0285 [hep-ph].

[10] L. J. Dixon and Y. Li, Bounding the Higgs Boson Width Through Interferometry, Phys.Rev.Lett. 111 (2013) 111802, arXiv:1305.3854 [hep-ph].

[11] J. M. Campbell, R. K. Ellis, and C. Williams, Bounding the Higgs width at the LHC using full analytic results for $\mathrm{gg} \rightarrow 2 \mathrm{e} 2 \mu$, arXiv: 1311.3589 [hep-ph] .

[12] J. M. Campbell, R. K. Ellis, and C. Williams, Bounding the Higgs width at the LHC: complementary results from $\mathrm{H} \rightarrow \mathrm{WW}$, arXiv:1312.1628 [hep-ph] .

[13] CMS Collaboration, S. Chatrchyan et al., Measurement of the properties of a Higgs boson in the four-lepton final state, Phys.Rev. D89 (2014) 092007, arXiv: 1312.5353 [hep-ex] .

[14] ATLAS Collaboration, G. Aad et al., Measurements of Higgs boson production and couplings in diboson final states with the ATLAS detector at the LHC, Phys.Lett. B726 (2013) 88-119, arXiv:1307.1427 [hep-ex]. 
[15] LHC Higgs Cross Section Working Group Collaboration, A. David et al., LHC HXSWG interim recommendations to explore the coupling structure of a Higgs-like particle, arXiv: 1209.0040 [hep-ph].

[16] G. Passarino, NLO Inspired Effective Lagrangians for Higgs Physics, Nucl.Phys. B868 (2013) 416-458, arXiv:1209.5538 [hep-ph].

[17] B. Grzadkowski, M. Iskrzynski, M. Misiak, and J. Rosiek, Dimension-Six Terms in the Standard Model Lagrangian, JHEP 1010 (2010) 085, arXiv: 1008.4884 [hep-ph ] .

[18] LHC Higgs Cross Section Working Group Collaboration, S. Heinemeyer et al., Handbook of LHC Higgs Cross Sections: 3. Higgs Properties, arXiv:1307.1347 [hep-ph] .

[19] R. Contino, M. Ghezzi, C. Grojean, M. Muhlleitner, and M. Spira, Effective Lagrangian for a light Higgs-like scalar, JHEP 1307 (2013) 035, arXiv: 1303.3876 [ hep-ph] .

[20] M. B. Einhorn and J. Wudka, The Bases of Effective Field Theories, Nucl.Phys. B876 (2013) 556-574, arXiv:1307.0478 [hep-ph].

[21] B. Dumont, S. Fichet, and G. von Gersdorff, A Bayesian view of the Higgs sector with higher dimensional operators, JHEP 1307 (2013) 065, arXiv:1304.3369 [hep-ph] .

[22] E. E. Jenkins, A. V. Manohar, and M. Trott, On Gauge Invariance and Minimal Coupling, JHEP 1309 (2013) 063, arXiv:1305.0017 [hep-ph] .

[23] K. Cranmer, S. Kreiss, D. Lopez-Val, and T. Plehn, A Novel Approach to Higgs Coupling Measurements, arXiv:1401.0080 [hep-ph] .

[24] J. Bain, Emergence in effective field theories, Eur.J.Phil.Sci. 3 (2013) no. 3, 257-273.

[25] S. Actis, A. Ferroglia, M. Passera, and G. Passarino, Two-Loop Renormalization in the Standard Model. Part I: Prolegomena, Nucl.Phys. B777 (2007) 1-34, arXiv : hep-ph/ 0612122 [hep-ph].

[26] S. Actis and G. Passarino, Two-Loop Renormalization in the Standard Model Part II: Renormalization Procedures and Computational Techniques, Nucl.Phys. B777 (2007) 35-99, arXiv:hep-ph/0612123 [hep-ph].

[27] S. Actis and G. Passarino, Two-Loop Renormalization in the Standard Model Part III: Renormalization Equations and their Solutions, Nucl.Phys. B777 (2007) 100-156, arXiv: hep-ph/0612124 [hep-ph].

[28] J. Elias-Miro, C. Grojean, R. S. Gupta, and D. Marzocca, Scaling and tuning of EW and Higgs observables, arXiv:1312.2928 [hep-ph].

[29] S. Willenbrock and C. Zhang, Effective Field Theory Beyond the Standard Model, arXiv:1401.0470 [hep-ph].

[30] C. Grojean, E. E. Jenkins, A. V. Manohar, and M. Trott, Renormalization Group Scaling of Higgs Operators and $\Gamma(\mathrm{H} \rightarrow \gamma \gamma)$, JHEP 1304 (2013) 016, arXiv: 1301.2588 [hep-ph ] .

[31] R. Alonso, E. E. Jenkins, A. V. Manohar, and M. Trott, Renormalization Group Evolution of the Standard Model Dimension Six Operators III: Gauge Coupling Dependence and Phenomenology, JHEP 1404 (2014) 159, arXiv: 1312 . 2014 [hep-ph] . 